\title{
Retrocausal model of reality for quantum fields
}

\author{
Peter D. Drummond ( 1 and Margaret D. Reid $\odot$ \\ Centre for Quantum and Optical Science, Swinburne University of Technology, Melbourne 3122, Australia \\ and Weizmann Institute of Science, Rehovot 7610001 Israel
}

(Received 28 August 2019; revised 12 March 2020; accepted 11 June 2020; published 19 August 2020)

\begin{abstract}
We show that one may interpret physical reality as random fields in space-time. These have a probability given by the expectation of a coherent state projection operator, called the $Q$-function. The resulting dynamical evolution includes retrocausal effects. This suggests that a physical universe exists without requiring observers, but with a well-defined probability for its field configuration. By including the meter dynamics, we show that field trajectories have quantum measurement properties without wave-function collapse, including sharp measured eigenvalues. We treat continuous and discrete measurements and show that this model predicts Bell inequality violations for measurements on correlated spins. A discussion is give of a number of well-known quantum paradoxes, showing how these can be treated in a realistic model of measurement. Our theory resolves a number of practical and philosophical issues in quantum measurement, and we compare it with earlier theories.
\end{abstract}

DOI: 10.1103/PhysRevResearch.2.033266

\section{INTRODUCTION}

The apparent quantum divide between macroscopic and microscopic worlds has never been more important. Experimentalists now probe systems of ever larger size [1,2], yet more deeply quantum properties [3]. It is commonplace for theoretical cosmologists to adopt the view of Coleman [4,5] and others [6], that the entire universe is quantum mechanical. Such progress leaves the paradoxes of the Copenhagen interpretation of quantum mechanics and the Schrödinger cat [7] in a central position in physics. Yet, to use the standard postulates of measurement theory [8], one must make a fundamental division between the microscopic and macroscopic. This is not accepted by those who see the universe as undivided [9] and is inconsistent with quantum cosmology.

Here we show that an ontological model of physical reality is obtainable using a mapping to a phase space of stochastic fields. The probability of a configuration is given by the $Q$ function of quantum field theory. As in statistical mechanics, any configuration has some probability to occur, but only one exists. The theory includes vacuum fluctuations and is frame invariant. This resolves paradoxes of quantum measurement $[7,10,11]$, since no separate observer and system is needed. We demonstrate that the introduction of a model for the meter generates measurement predictions identical to the usual ones.

The underlying fields provide an ontological model of both macroscopic and microscopic realism [12-14]. This can be applied to the physical universe as a whole. No explicit projection is needed to obtain sharply defined eigenvalues, although

Published by the American Physical Society under the terms of the Creative Commons Attribution 4.0 International license. Further distribution of this work must maintain attribution to the author(s) and the published article's title, journal citation, and DOI. one must include the physics of measurement. This eliminates the observer-dependent wave-function collapse criticized by many physicists $[15,16]$. To demonstrate this, we study measurement models of discrete and continuous measurements. We prove that Bell inequalities [17] are violated in this model of reality.

In our approach, we accept the principle that measurement is central to interpreting quantum theory. To measure a spin projection, one must include the polarizer. Until one does this, the spin wave-function is an incomplete description. One can describe a system with or without a meter. However, the results may change, as Bohr emphasized [18]: The meter is part of the universe.

Foundational problems in quantum mechanics have been the subject of much study, with recent claims $[19,20]$ that the wave-function is ontological [21-23]. In these works, reality is defined as what is prepared in a laboratory, with causality proceeding from past to future.

We regard this as too narrow a definition. Physical reality surely exists in all space-time, so that the most viable ontologies are space-time fields. In our approach, stochastic fields in space-time are real objects. This permits the theory to have a statistical interpretation $[23,24]$. Theorems requiring an ontological wave-function do not apply to our model [23] because of retrocausality [25-30] due to negative diffusion terms in the dynamical equations [31].

Previous models include the de Broglie-Bohm and related theories with both wave-function and particle coordinates $[9,32]$, discrete models used in quantum information [33,34], and a nonrelativistic phase-space model with an epistemic restriction [35]. Other theories include consistent [36] or decoherent histories [37-39], and quantum Darwinism [40]. These are related to relative state or "many-worlds" theories [41] which depend on observers.

By comparison, our model applies to quantum fields, is compatible with relativity, and includes particle statistics. We 
define elements of reality as fields in space-time, which exist at all times. These satisfy an action principal with past and future boundary conditions. This approach fulfills Einstein's requirements [42] that it is complete, formulated in terms of space-time fields, and objective, without needing observers. In simple terms, we generate a definite probability for obtaining one universe, which gives a well-defined, objective procedure for measurement and quantum models of the universe.

In our model, the only requirement to understand measurement is the inclusion of a meter. Our use of a meter for this purpose follows Bohr and Bell $[18,43]$. No additional decoherence mechanism [44-46] or nonlinearity [47] is necessary to achieve this.

\section{A STOCHASTIC FIELD REALITY MODEL}

The question of what is the reality that quantum theory describes was raised by Bohr, Einstein, Born, and others. It was the subject of much debate at the Solvay conferences [48]. In this section a reality model for quantum theory is proposed using stochastic fields.

\section{A. Definitions of reality}

The definition of reality most common in quantum mechanics uses Bohr's interpretation [18]. This claims that one can only speak of reality in terms of macroscopic physical measurements. To obtain measured results, the elements of microscopic reality, if they exist, must be amplified to macroscopic levels. To quote Bohr [49]: "The unambiguous account of proper quantum phenomena must, in principle, include a description of all relevant features of the experimental arrangement."

In his Autobiographical Notes, Einstein [42] makes the following remark: "There is no such thing as simultaneity of distant events; consequently there is also no such thing as immediate action at a distance in the sense of Newtonian mechanics." From this, he concluded: "It therefore appears unavoidable that physical reality must be described in terms of continuous functions in space."

This question of the existence of an objective reality was also raised by Max Born, in his Nobel lecture [50]. In view of difficulties in defining a simultaneous particle position and momentum, Born asked "What is the reality which our theory has been invented to describe?" He suggested that physical reality might have a more abstract basis: "For this, well-developed concepts are available which appear in mathematics under the name of invariants in transformations. Every object that we perceive appears in innumerable aspects."

If one accepts Einstein's viewpoint, then one should define reality as an objective, continuous set of events, defined locally in space and time. By objective, we mean that the events do not require an observer for their existence. However, according to Bohr, if observers are present, then one must not expect reality to be independent of them, either. In observing a system, an interaction occurs which modifies both system and observer.

We propose a definition of reality that unifies all three viewpoints. Reality should exist in space and time, have a mathematical expression in terms of invariants, and agree with quantum predictions of macroscopic measurements. This does not mean that reality ceases to exist or is undefined in the absence of measurement. As elements of reality, our approach uses stochastic fields which exist at all times, in the future as in the past, to fulfill Einstein's requirements. To calculate probabilities, we use the $Q$-function of quantum mechanics, which is a positive and unique distribution that exists for all quantum states. It is interpreted as the probability for a particular field configuration defined at a given time.

\section{B. The stochastic field probability}

We define the probability of a particular realization $\lambda$ at time $t$ as the positive definite function $Q$, where

$$
Q(\lambda, t)=\operatorname{Tr}[\hat{\rho}(t) \hat{\Lambda}(\lambda)] .
$$

The projector $\hat{\Lambda}$ is normalized such that $\int \hat{\Lambda}(\lambda) d \lambda=\hat{1}$, which means that the distribution is normalized so that

$$
\int Q(\lambda) d \lambda=1
$$

The configurations $\lambda$ are representations of the Lie groups compatible with the commutation relations, reflecting Born's remark about invariants. We define $\lambda=[\boldsymbol{\psi}, \boldsymbol{\xi}]$ where $\boldsymbol{\psi}=$ $\left[\psi_{1}, \psi_{2} \ldots\right]$ are fields representing bosons. Since the physical universe comprises fermions and bosons, we include a fermionic representation as well [51,52]. Here $\boldsymbol{\xi}=$ $\left[\xi_{1}, \xi_{2}, \ldots\right]$ are real antisymmetric matrices representing fermions, and $\hat{\Lambda}(\boldsymbol{\lambda})=\prod_{b, f} \hat{\Lambda}_{b}\left(\boldsymbol{\psi}_{b}\right) \hat{\Lambda}_{f}\left(\xi_{f}\right)$ is a Gaussian, positive-definite [53] operator for bosons $(b)$ and fermions $(f)$.

For simplicity, in this paper we construct $Q$-functions from coherent state projectors. We first expand the operator field $\hat{\psi}(\boldsymbol{r})$ in annihilation and creation operators, $\hat{a}_{n}$ and $\hat{a}_{n}^{\dagger}$, where $\left[\hat{a}_{n}, \hat{a}_{m}^{\dagger}\right]=\delta_{n m}$. The coherent state approach involves stochastic fields directly, since in this case one can define

$$
\hat{\Lambda}_{b}(\boldsymbol{\psi})=\frac{1}{\pi^{M}}|\boldsymbol{\alpha}\rangle_{c}\left\langle\left.\boldsymbol{\alpha}\right|_{c}\right.
$$

Here $M$ is the number of spatial and spin modes in the bosonic field expansion, and the coherent states $|\boldsymbol{\alpha}\rangle_{c}$ are defined [53] as follows:

$$
|\boldsymbol{\alpha}\rangle_{c}=e^{\boldsymbol{\alpha} \cdot \hat{\boldsymbol{a}}^{\dagger}-|\boldsymbol{\alpha}|^{2} / 2}|\mathbf{0}\rangle .
$$

The field $\boldsymbol{\psi}(\boldsymbol{r})$ is obtained from $\hat{\boldsymbol{\psi}}(\boldsymbol{r})$ by replacing the annihilation and creation operators operators with complex amplitudes $\alpha_{n}$ and $\alpha_{n}^{*}$, respectively. For a Hermitian quantum field $\hat{\psi}(\boldsymbol{r})$, the corresponding element of reality is

$$
\boldsymbol{\psi}(\boldsymbol{r})=\sum_{n=1}^{M}\left[\boldsymbol{u}_{n}(\boldsymbol{r}) \alpha_{n}+\boldsymbol{u}_{n}^{*}(\boldsymbol{r}) \alpha_{n}^{*}\right],
$$

where $\boldsymbol{u}_{n}(\boldsymbol{r})$ are $M$ normalized spatial mode functions. This expansion is directly applicable to electromagnetic modes, which will be used to illustrate the properties of measurements. As a result, we will consider $\lambda$ as comprising a set of $M$ mode amplitudes $\boldsymbol{\alpha}$ in the examples described here. These can be converted into $2 M$ real quadrature amplitudes, $\boldsymbol{q}=\boldsymbol{\alpha}+\boldsymbol{\alpha}^{*}$ and $\boldsymbol{p}=\left(\boldsymbol{\alpha}-\boldsymbol{\alpha}^{*}\right) / i$.

Massive particles including fermions and bosons in the QCD Higgs sector require more sophisticated methods 
involving Gaussian functions of field operators, which are described elsewhere [51,52,54-57]. Antisymmetric matrices $\xi$ can be transformed into a matrix form equivalent to real and anomalous correlations of fermions. These in turn can be reduced to products of space-time fields. A similar method can be used for bosons with symmetric real matrices. This establishes a link with the symmetry properties and invariants of the group-theoretic homogeneous spaces of Cartan [58] and Hua [59].

The $Q$-function is the probability at some time $t$, but in this model, fields have continuous trajectories $\lambda(t)$ defined at all times. Before giving a detailed analysis, we note that our proposal is not ruled out by no-go theorems $[60,61]$ for phase-space models, which claim that they cannot be realistic theories. These theorems do not allow for the presence of meters in a measurement.

The necessity of including the meter in an analysis of macroscopic measurements implies that a theory that leaves out the physics of the meter is unrealistic. Omitting meters leads to relatively large vacuum fluctuations. We show below that the inclusion of the meter-regarded as an amplifier-eliminates this problem. Quantum fluctuations become asymptotically negligible compared to the measured eigenvalue, in the large gain limit. In this sense our model of reality is contextual [62], since the meter changes what is measured due to retrocausality.

\section{Uniqueness}

We next consider the question of uniqueness. There is no one-to-one correspondence between a phase-space variable and a quantum state. A stochastic phase-space coordinate $\boldsymbol{\alpha}$ does not uniquely define a quantum state, and nor does the quantum state uniquely define $\boldsymbol{\alpha}$. Rather, a quantum state is defined by a distribution $Q(\boldsymbol{\alpha})$ for $\boldsymbol{\alpha}$. If one regards quantum states as elements of reality [21], then the lack of a unique mapping between quantum states and the phase-space coordinates used here may seem to be a problem.

However, in any measurement of a quantum state, where the state is not an eigenstate of the measurement operator, random results occur. Since even random measurement results are real, one must have an explanation of these. They could be thought to originate from the measurement apparatus, but this gives an infinite recursion, where larger and larger quantum states are required. For this reason, it is not unreasonable to suppose that the quantum state is an incomplete description, just as in the ensembles of statistical mechanics.

Our conclusion is that the originators of quantum mechanics regarded the quantum wave-function as statistical [50] for good reason. A one-to-one mapping of states to reality is not necessary. There are many possible phase-space variables that can occur in a given quantum state, consistent with a statistical picture. This is consistent with the fact that quantum measurements give random results at the level of Planck's constant, apart from the case where an eigenstate is prepared. Even this may have microscopic randomness, as we show later.

There is a second question concerning uniqueness. In the single-mode case one may consider an eigenstate for an observable $\hat{q}=\hat{a}+\hat{a}^{\dagger}$, with eigenvalue $q_{1}$. Another eigenstate

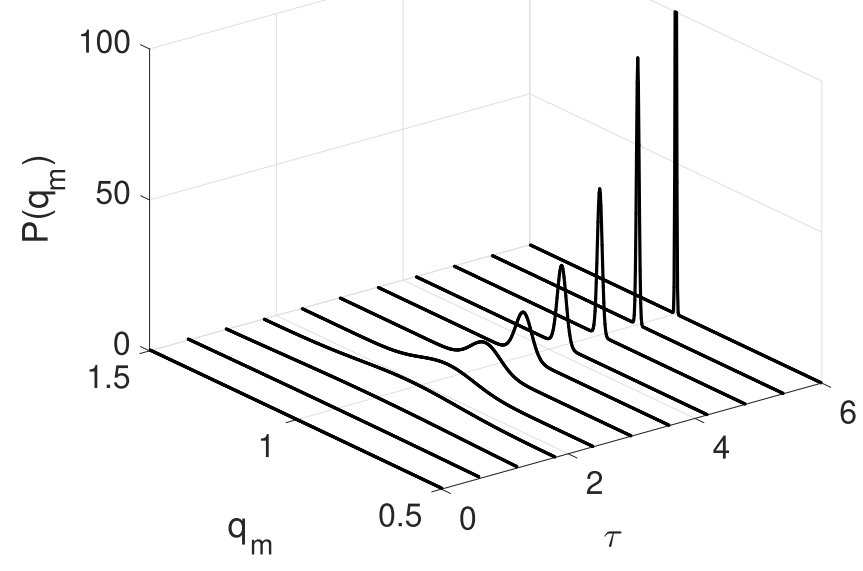

FIG. 1. Probability density $P\left(q_{m}\right)$ as a function of the measured quadrature amplitude $q_{m}$ and dimensionless time $\tau=g t$ for a $q$ eigenstate with $q_{0}=1$. The increasing sharpness with gain shows how a continuous eigenvalue can be recovered despite vacuum noise. The vertical scale is truncated at $P\left(q_{m}\right)=100$ for visibility.

could have eigenvalue $q_{2}$. Because of the width of the $Q$ function for each eigenstate, the same $\alpha$ could correspond to either outcome for $\hat{q}$. A given $\alpha$ therefore does not correspond to a unique eigenvalue for a measurement outcome. We explain below how this is consistent with the requirement of time-symmetric boundary conditions, which give unique measurement outcomes for eigenstates, as shown in Fig. 1.

\section{Alternative phase-space representatives}

There are many quasiprobabilities for quantum fields, in both bosonic and fermionic cases: why should one choose the $Q$-function? The Wigner representation [63] was proposed as a statistical theory by Moyal [64], but is nonpositive, and has no direct probabilistic interpretation $[65,66]$. Another possibility, the Glauber $P$-function [53], is nonpositive and highly singular for some quantum states. There is a continuous class of " $s$-ordered" bosonic quasiprobabilities [67]. The case of $s=-1$ is the $Q$-function, which is the most compact of the s-ordered distributions that are always positive. The positive $P$-function has a distribution which is positive and nonsingular. Since it is not unique [68], it does not give a distinctive model.

In the fermionic case, there is a positive $Q$-function which we use. There are also Grassman-valued $P$-distribution quasiprobabilities [69]. However, these have no probabilistic interpretation. As in the bosonic case, there are $P$-distributions using expansions in fermionic Gaussian states. These are either not unique [55,56,70] or else not always positive [71].

In summary, in our approach to the measurement problem we require that the theory has predictions equivalent to quantum mechanics. It should be unique, and have a positive probability distribution. Each possible real outcome that can occur must have a well-defined dynamical equation. A single trajectory of stochastic fields defined in space-time then corresponds to a possible universe. This resembles the way that 
statistical mechanics describes an ensemble: There are many possible outcomes, but only one is realized.

The generalized $Q$-function distribution for stochastic fields [52,72] satisfies our requirements for an ontology underlying quantum mechanics. Other phase-space representations, like the Wigner representation, are either nonpositive or nonunique. We will show that the fact that the $Q$-function phase-space trajectory is determined by future as well as past boundary conditions addresses issues associated with nonorthogonality of coherent states. This results in measurement outcomes that correspond to experiment.

\section{STOCHASTIC FIELD DYNAMICS}

$Q$-function distributions also include vacuum fluctuations. We regard these as real, since the fields are ontological. From the Heisenberg uncertainty principle, the $Q$-function is not infinitely sharp, leading to restrictions on physically valid distributions. Yet eigenvalues of Hermitian operators do exist. In fact, these sharply defined outcomes are fundamental to quantum mechanics. To understand this issue, we analyze measurements.

To treat measurement dynamics, one can make use of several techniques for analyzing $Q$-function time-evolution. Distributions can be calculated using the time-evolution of $\hat{\rho}(t)$, which follows standard techniques. It is also instructive to follow individual trajectories as realistic dynamical histories. Here, we present a summary of this issue, by remarking that the probability distribution obeys a first-order differential equation in time. This dynamical equation is obtained uniquely and exactly from equivalence theorems and identities that map quantum operators into differential operators.

\section{A. Generalized Fokker-Planck equations}

Unitary dynamics is given in quantum field theory by the standard result that $i \hbar \partial_{t} \hat{\rho}=[\hat{H}, \hat{\rho}]$, where we use the notation that $\partial_{t} \equiv \partial / \partial t$. This implies that the probability distribution $Q(\lambda, t)$ evolves according to

$$
\partial_{t} Q(\lambda, t)=\frac{i}{\hbar} \operatorname{Tr}\{[\hat{H}, \hat{\Lambda}(\lambda)] \hat{\rho}(t)\} .
$$

Operator expressions occurring inside the trace, of form $\hat{H} \hat{\Lambda}$ or $\hat{\Lambda} \hat{H}$, can then be reduced to differential expressions in the phase space. In the single-mode bosonic case, defining $\partial_{\alpha} \equiv$ $\partial / \partial \alpha$, the following differential identities are well known:

$$
\begin{aligned}
\hat{a}^{\dagger}|\alpha\rangle_{c}\left\langle\left.\alpha\right|_{c}\right. & =\left[\partial_{\alpha}+\alpha^{*}\right]|\alpha\rangle_{c}\left\langle\left.\alpha\right|_{c},\right. \\
\hat{a}|\alpha\rangle_{c}\left\langle\left.\alpha\right|_{c}\right. & =\alpha|\alpha\rangle_{c}\left\langle\left.\alpha\right|_{c} .\right.
\end{aligned}
$$

Examples of these mappings to obtain unitary $Q$-function dynamics are given in the next section. Using these and other operator identities as required, this is equivalent to a zero-trace generalized diffusion equation for the elements of reality $\lambda$, of form

$$
\dot{Q}(\lambda, t)=\mathcal{L}(\lambda) Q(\lambda, t) .
$$

The resulting differential equation has a diffusive character if the differential terms are no higher than second order. Such terms are found in the case of Hamiltonian evolution with no more than quartic nonlinearities in the fields. This covers the most important fundamental quantum field theories, including quantum electrodynamics. This algebraic restriction is compatible with quantum field Hamiltonians, but not particle Hamiltonians in first quantization. Therefore, these methods apply to quantum fields, not quantum particles.

The dynamical equation for $Q$ is a generalized FokkerPlanck equation. It has an action principle with both past and future boundary conditions [73]. The dynamics is equivalent to quantum mechanics, so it is consistent with known physical observations. Generalized Fokker-Planck equations are not the same as the dissipative equations that describe coupling to reservoirs. In particular, they have a zero-trace diffusion matrix for unitary evolution, which leads to a retrocausal interpretation.

Although there are similarities with dissipative equations, unitary evolution under a Hamiltonian is reversible. The corresponding $Q$-function dynamical equations have welldefined solutions whether boundary conditions are specified in the past or the future. This occurs because of second order derivative terms which are not positive definite. The dynamical equations for the distribution are therefore not FokkerPlanck equations. There is also no conventional stochastic equation to describe an individual trajectory.

This issue of the existence of a stochastic equation is immaterial if one regards the entire distribution as a single element of reality. From this perspective, an individual phasespace coordinate, whether a field or a complex amplitude, is only a part of the wave-function or distribution. However, since we take the view that an individual stochastic field is an element of reality, its trajectory is important. This satisfies a real action principle equivalent to a forwards-backwards stochastic equation, propagating in both time directions.

To give more detail, consider a generalized Fokker-Planck dynamics. Without loss of generality, we can use real phasespace variables $\phi^{\mu}$, such that

$$
\dot{Q}=\partial_{\mu}\left[-A^{\mu}+\frac{1}{2} \partial_{\nu} D^{\mu \nu}\right] Q .
$$

For simplicity, we assume that $D^{\mu \nu}=D^{\mu} \delta^{\mu \nu}$ is diagonal and constant, which is valid for the amplifier examples used here. For $M$ modes in bosonic cases, the $2 M$ variables are divided into positive and negative diffusion terms, $\boldsymbol{\phi}=[\boldsymbol{p}, \boldsymbol{q}]$, such that if $\mu \leqslant M$ then $D^{\mu}>0$; otherwise $D^{\mu}<0$. The $q$ and $p$ are real, complementary field quadrature amplitudes, not mechanical position and momenta coordinates.

By contrast, a conventional dissipative Fokker-Planck equation has $D^{\mu}>0$ for all $\mu$ [74]. This is typically obtained by including a large number of reservoir degrees of freedom, together with other approximations. We emphasize that there is no reservoir required here. The present result is obtained directly from the Hamiltonian, and there is no dissipation. Hence, this method is fundamental, provided there are no higher-order nonlinearities than quartic. Dissipative reservoirs can also be included if required.

There is a corresponding real path integral that defines the possible trajectories of the solutions, which is

$$
Q\left(\boldsymbol{\phi}_{f}, t_{f} \mid \boldsymbol{\phi}_{0}, t_{0}\right) \propto e^{-\int \mathcal{L}(\boldsymbol{\phi}, \dot{\boldsymbol{\phi}}) d t} .
$$

This is different to a Feynman path integral which gives complex amplitudes, with paths that have no realistic 
interpretation. Here the Lagrangian is real, and there is no imaginary coefficient. For constant diffusion, the Lagrangian density has the form

$$
\mathcal{L}=\frac{1}{2} \sum_{\mu}\left\{\frac{1}{d^{\mu}}\left(\dot{\phi}^{\mu}-A^{\mu}\right)^{2}-s_{\mu} \partial_{\mu} A^{\mu}\right\},
$$

where $d^{\mu}=\left|D^{\mu}\right|$ and $s_{\mu}=\operatorname{Sign}\left(D^{\mu}\right)$. The path integral boundary conditions for $\boldsymbol{p}$ are fixed in the past and open in the future, while those for $\boldsymbol{q}$ are fixed in the future and open in the past.

This is equivalent to a forwards-backwards stochastic equation, with Gaussian $\delta$-correlated noises $\left[\boldsymbol{w}_{p}, \boldsymbol{w}_{q}\right]$ such that $\left\langle\dot{w}^{\mu}(t) \dot{w}^{\nu}\left(t^{\prime}\right)\right\rangle=\left|D^{\mu \nu}\right| \delta\left(t-t^{\prime}\right)$. The relevant equations are as follows:

$$
\begin{aligned}
& \boldsymbol{p}(t)=\boldsymbol{p}\left(t_{0}\right)+\int_{t_{0}}^{t} \boldsymbol{A}_{p}\left(t^{\prime}\right) d t^{\prime}+\int_{t_{0}}^{t} d \boldsymbol{w}_{p}, \\
& \boldsymbol{q}(t)=\boldsymbol{q}\left(t_{f}\right)-\int_{t}^{t_{f}} \boldsymbol{A}_{q}\left(t^{\prime}\right) d t^{\prime}-\int_{t}^{t_{f}} d \boldsymbol{w}_{q} .
\end{aligned}
$$

$Q$-functions are real probability distributions, and one can interpret an individual path as a possible stochastic trajectory. From Eq. (12), trajectory boundary values must be specified both in the past and in the future, depending on the quadrature, which is different to having initial conditions only.

\section{B. Boundary conditions}

The boundary conditions are uniquely constrained by the quantum state. These distributions, specified in the past and the future, must lead dynamically to the initial distribution $Q\left(\boldsymbol{\phi}, t_{0}\right)$, which is related through Eq. (1) to the required initial quantum state. This is a subtle issue, and we describe it in detail in this subsection.

In quantum mechanics, the assumption is generally made that any quantum state can be generated on demand. For simplicity, we make this assumption also, and in this respect our approach is like the usual treatment. In practice, experimental state preparation is nontrivial. Conditional state preparation is often necessary when high fidelities are required [75]. Thus, there are quantum states where preparation on demand is far from easy, and this requires discussion, given the approach to reality described here.

For example, a common way to prepare a specific field quadrature is through an EPR experiment [76] in which two correlated quadratures are prepared through down-conversion. The specification of the eigenvalue may not be known until a future time measurement is made of the correlated quadrature. This can be made in the future, and viewed as a future-time boundary value. A careful analysis suggests that this is not uncommon. Future boundary conditions are implicit in the analysis of Maxwell's equations for radiating fields [26]. They are discussed for both classical [27] and quantum fields [29] and are as fundamental as past time boundary conditions.

The existence of a quantum state or density matrix $\hat{\rho}\left(t_{0}\right)$ at a given time $t_{0}$ is equivalent to having a well-defined distribution $Q\left(\boldsymbol{\phi}, t_{0}\right)$. Yet elements of reality must exist both in the past and future, so that they are defined in all reference frames. Given the equations above, the dynamics of any phase-space coordinate $\boldsymbol{\phi}$ requires probabilities of initial conditions for forward-time components $\boldsymbol{p}$, and probabilities of final conditions for components $\boldsymbol{q}$ that propagate to the past.

This approach appears to differ from everyday experience. A common philosophy is that causality must be unidirectional, but this is essentially a classical world-view. It is usually expected that one may prepare any required quantum state, which propagates to the future. However, retrocausal dynamics requires the specification of both initial and final conditions. To obtain a given $\hat{\rho}\left(t_{0}\right)$, these boundary conditions on $\boldsymbol{\phi}$ and their distributions in the future and past must therefore dynamically give rise to $Q\left(\boldsymbol{\phi}, t_{0}\right)$ at the required time $t_{0}$.

In summary, an element of reality requires the specification of both future and past time boundary values for its dynamics.

\section{STOCHASTIC MEASUREMENT MODELS}

In quantum mechanics, it is a standard postulate that any state $\hat{\rho}$ can be experimentally prepared, and any Hermitian operator $\hat{O}$ can be measured. However, Bohr was careful to insist that in the case of measurement, one has to consider the experimental apparatus used to obtain the macroscopic output measured $[18,48]$. State preparation and measurement are complementary, and state preparation therefore requires an operational definition as well, as discussed above.

Here we will focus on the operational measurement issue, by considering an example of a single bosonic mode, with $\alpha=(q+i p) / 2$. The normalized projector, $\hat{\Lambda}_{b}$ is

$$
\hat{\Lambda}_{b}=\frac{1}{\pi}|\alpha\rangle_{c}\left\langle\left.\alpha\right|_{c} .\right.
$$

For example, in a vacuum state $|0\rangle$, the distribution has a finite variance due to vacuum fluctuations, given by

$$
Q(\alpha)=\frac{1}{\pi} e^{-|\alpha|^{2}} .
$$

Yet it is not just the vacuum state that has a finite variance. In fact, all states have substantial quantum fluctuations in this approach. The most puzzling question to be answered with a stochastic field interpretation of reality is therefore what this implies in measurement terms. How can real quantum fluctuations be compatible with the fact that there are quantum states with well-defined eigenvalues?

\section{A. Measurement of a continuous eigenvalue}

To understand this, we will treat the theory of measurement in an idealized case. For simplicity, we expand the quantum fields in mode operators. We first treat a model for the measurement of a continuous variable, the $\hat{q}$ quadrature of the single-mode radiation field. Here, we consider the simplest theory for a measurement of $\hat{q}$, corresponding to a measurement on the system by a meter, where the system observable of interest is directly amplified using a low-noise parametric amplifier [77-79]. These are widely used practical devices in modern quantum technology [80-82], and epitomize how one makes quantum-limited field quadrature measurements in the laboratory. Such technologies are also used for quantum limited position measurements [83-85].

We let $\hat{q}=\hat{a}+\hat{a}^{\dagger}$ and $\hat{p}=\left(\hat{a}-\hat{a}^{\dagger}\right) / i$ be the field quadratures of mode $\hat{a}$. The amplification takes place via interaction of the system with a meter. In our model, the interaction 
Hamiltonian in the rotating frame of our meter is that given by a parametric amplifier:

$$
\widehat{H}=\frac{i \hbar g}{2}\left[\hat{a}^{\dagger 2}-\hat{a}^{2}\right] .
$$

We can obtain the evolution of the system and meter by solving for the quantum state or by using the Heisenberg equations, which have operator solutions with $\hat{q}(t)=\hat{q}(0) e^{g t}$ and $\hat{p}(t)=\hat{p}(0) e^{-g t}$. For the choice $g>0$, we see that $\hat{q}$ is amplified, as required if we are to view the meter as a measuring device for $\hat{x}$. Equivalently, it is also possible to use the $Q$-function Fokker-Planck or stochastic equations, which we explain below. Given an initial vacuum state, in which $\left\langle\Delta \hat{q}^{2}(0)\right\rangle=\left\langle\Delta \hat{p}^{2}(0)\right\rangle=1$, the $\hat{p}$ quadrature becomes squeezed as time evolves, with the variance $\left\langle\Delta \hat{p}^{2}(t)\right\rangle$ reducing below the vacuum level, while the $\hat{q}$ quadrature develops a large variance.

Suppose the quantum system is prepared in a superposition of eigenstates $\left|q_{0}\right\rangle_{x}$ of the $\hat{q}$ quadrature, each with eigenvalue $q_{0}$, and a total variance $\left\langle\Delta \hat{q}^{2}(0)\right\rangle$. The parametric amplifier amplifies the $\hat{q}$ quadrature to a macroscopic level. After measurement, if the gain is $G(\tau)=e^{\tau}$, where $\tau \equiv g t$ is the dimensionless time, the resulting variances in the phase-space variables are

$$
\begin{aligned}
& \left\langle\Delta q^{2}(t)\right\rangle=1+G^{2}\left\langle\Delta \hat{q}^{2}(0)\right\rangle, \\
& \left\langle\Delta p^{2}(t)\right\rangle=1+\left\langle\Delta \hat{p}^{2}(0)\right\rangle / G^{2} .
\end{aligned}
$$

The vacuum noise contribution to the ontological phase-space variable $q(\tau)$ has a variance of unity, even in an eigenstate of $\hat{q}$ where $\left\langle\Delta \hat{q}^{2}(0)\right\rangle=0$. Yet experimentalists can identify a reproducible eigenvalue $q_{0}$ after the measurement. This is possible, because the measured estimate of the eigenvalue is $q_{m}=q(\tau) / G(\tau)$. The variance in $q(\tau)$-regarded as a real event-is now dominated by $\left\langle\Delta \hat{q}^{2}(0)\right\rangle$. After measurement, the phase-space variable $q$ is the actual result of measuring the $\hat{q}$ quadrature, once the gain $G$ is accounted for. The measured value $q_{m}$ has a variance of $1 / G^{2}$ that vanishes in the asymptotic, large gain limit.

In the limit of an initial eigenstate, $|\psi(0)\rangle=\left|q_{0}\right\rangle_{x}$, we find using standard techniques from quantum theory that after measurement with dimensionless interaction time $\tau$, the marginal probability for a $Q$-function trajectory with measured eigenvalue $q_{m}=q(\tau) / G(\tau)$ is

$$
P\left(q_{m}, \tau\right)=\sqrt{\frac{G^{2}(\tau)}{2 \pi}} \exp \left[-\frac{1}{2} G^{2}(\tau)\left(q_{m}-q_{0}\right)^{2}\right] .
$$

This probability distribution is plotted in Fig. 1, demonstrating that the amplifier has the desired effect. It causes a relative narrowing in the probability distribution of measured outcomes. There is no sharp boundary imposed between "classical" and "quantum" worlds, no measurement projection, and not even any decoherence. Yet the outcome is rather clear. The effect of a quantum limited amplifier is to eliminate noise, leading to a well-defined outcome.

\section{B. $Q$-function dynamical equations}

The calculation given above used a standard quantum operator approach. One can calculate the result of the measurement more directly by using the phase-space dynamical

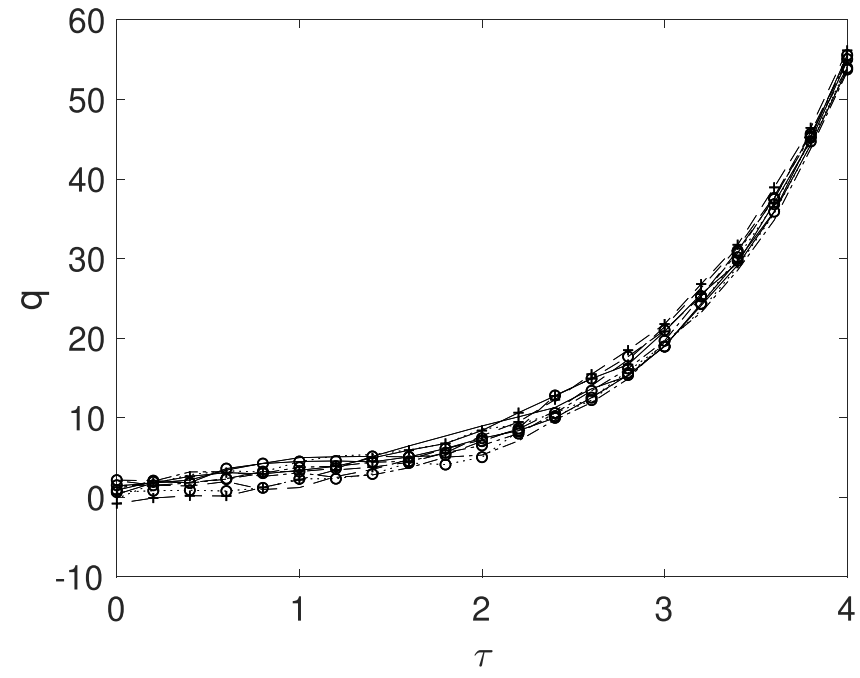

FIG. 2. Graph of 10 random trajectories of $q(\tau)$ vs. dimensionless time $\tau$, for retrocausal evolution under the amplification due to measurement of $\hat{x}$ on a system prepared in an eigenstate $\left|q_{0}\right\rangle$ of $\hat{q}$ at $\tau=0$. The dynamics for $q$ are solved backward in time, starting with the final condition $q\left(\tau_{f}\right)$. The trajectories for $q$ correspond to a near eigenstate of $\hat{q}$. The ensemble averaged noise at $\tau=0$ is the same as the final noise at $\tau=\tau_{f}$, with a variance in $q$ of 1 .

trajectories. We now consider the same measurement process using the $Q$-function dynamical equation,

$$
\frac{d Q}{d t}=-\frac{g}{2} \operatorname{Tr}\left\{\left[\hat{a}^{\dagger 2}-a^{2}, \hat{\Lambda}(\boldsymbol{\alpha})\right] \hat{\rho}\right\} .
$$

This can be written in terms of mode creation and annihilation operators using the identities given in Eq. (7). After making the substitutions of differential identities instead of operators, the dynamical evolution of the $Q$-function for unitary evolution is obtainable from the differential equation:

$$
\frac{d Q}{d t}=-\frac{g}{2}\left[\frac{\partial^{2}}{\partial \alpha^{2}}+2 \frac{\partial}{\partial \alpha} \alpha^{*}+h c\right] Q .
$$

We now make a variable change to real variables, so that $\alpha=$ $x+i p$, which gives

$$
\frac{d Q}{d \tau}=\left[\frac{\partial}{\partial p} p+\frac{\partial^{2}}{\partial p^{2}}-\left(\frac{\partial}{\partial q} q+\frac{\partial^{2}}{\partial q^{2}}\right)\right] Q .
$$

The $Q$-function equation for $q$ combines gain with negative diffusion, while that for $p$ is the exact time reversal or complement, since it combines loss with positive diffusion. This leads to time-reversed causation for the $q$ quadrature. The retrocausal evolution of the measured variable gives insight into why the vacuum fluctuations associated with the ontological phase-space variable $q$ for the $\hat{q}$ eigenstate, remain small during measurement of $q$. One might have expected these vacuum fluctuations to grow due to amplification, but this in fact does not happen, as shown by the trajectory graphs given in Fig. 2.

A simple way to explain this is that an eigenstate has the minimum possible level of quantum fluctuations in a stochastic field ontology. This noise level is not zero, as one might expect from the Heisenberg uncertainty principle. Instead, the $Q$-function noise remains at the level of Planck's constant, as 
shown by Eq. (16). The balance of noise and loss is such as to exactly maintain this level of quantum noise in the limit of a highly squeezed state. This behavior is similar to what occurs with gain, since gain is just time-reversed quantum squeezing.

However, this residual vacuum noise is not observable in macroscopic measurements. A measurement does not eliminate quantum noise, but rather reduces it to an arbitrarily low level relative to the observable. In a retrocausally amplified signal the relative noise becomes smaller with increased gain. This is simply the time-reversed corollary of the well-known physics of causally attenuated signals, where relative noise becomes larger with increased loss. The eigenvalue is obtained as the asymptotic limit of a high-gain measurement. This explains the distinction between microscopic and macroscopic behavior as a limiting process. There is no artificial boundary to be crossed.

Such retrocausal behavior is known to also provide a mechanism for overcoming the restrictions of Bell's theorem $[29,86]$, and the details of how this works in the present approach are given in the next section.

To summarize these results, the $Q$-function stochastic field quadrature after a measurement is $q=G q_{0}+\epsilon$, where $\epsilon$ is some random vacuum noise with $\left\langle\epsilon^{2}\right\rangle=1$. From the amplified macroscopic value $q$, the experimentalist infers a measured eigenvalue of $q_{m}=q_{0}+\epsilon / G$. A sharp eigenvalue $q_{0}$ is recovered from the measured data in the limit of an ideal, infinite gain meter, as $\tau \rightarrow \infty$, with no assumptions. The $Q$-function dynamical equations give the same prediction as we obtained with a standard quantum approach. One can also treat less idealized measurements with noise and decoherence, if required.

\section{Trajectory picture}

For unitary $Q$-function evolution equations, the diffusion matrix is traceless and equally divided into positive and negative definite parts. In this case the $p$ quadrature decays and has positive diffusion, while the the $q$ quadrature shows growth and amplification, but has negative diffusion in the forward time direction. The amplified quadrature, which corresponds to the measured signal of a parametric amplifier, propagates stochastically backward in time from the future.

To obtain a single, probabilistic trajectory, one must therefore constrain $q$ by a future boundary condition. For simplicity, we suppose the initial $Q$-function is factorizable. This is the case, for example, if the eigenstate is modeled as a Gaussian squeezed state in the limit of large quantum squeezing. Hence, the $Q$-function solutions can always be factorized as a product with $Q(\alpha, \tau)=P_{q}(q, \tau) P_{p}(p, \tau)$. Then, if $\tau_{-}=\tau_{f}-$ $\tau$ is the backward time direction, the time-evolution of each of these factors is quasidiffusive, with an identical diffusive structure, except that it occurs in each of two different time directions:

$$
\begin{aligned}
& \frac{d P_{q}}{d \tau_{-}}=\left[\frac{\partial}{\partial q} q+\frac{\partial^{2}}{\partial q^{2}}\right] P_{x}, \\
& \frac{d P_{p}}{d \tau}=\left[\frac{\partial}{\partial p} p+\frac{\partial^{2}}{\partial p^{2}}\right] P_{p} .
\end{aligned}
$$

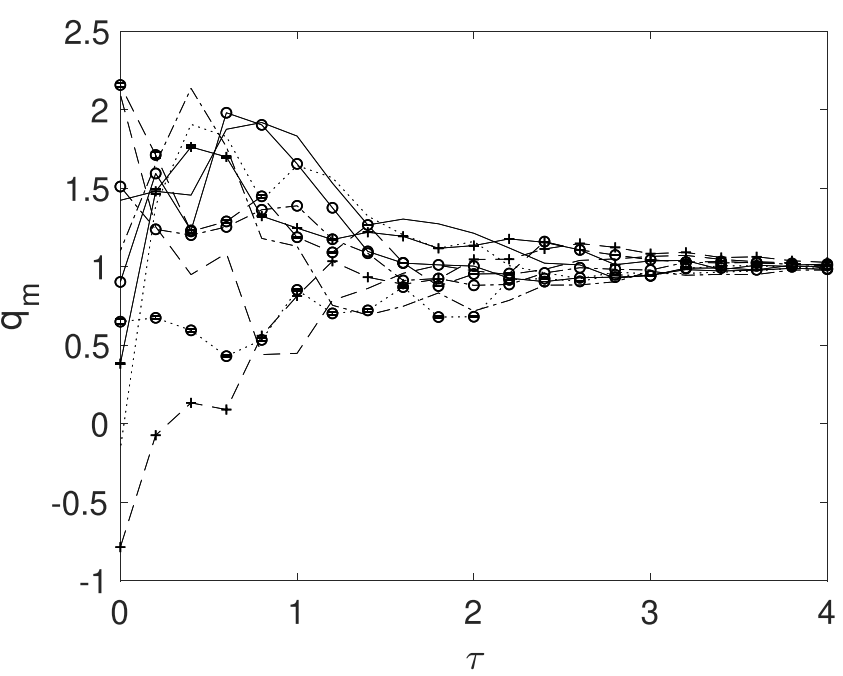

FIG. 3. Graph of 10 individual trajectories of the measured eigenvalue $q_{m}(\tau)=q(\tau) / G(\tau)$ vs. dimensionless time $\tau$, for evolution under the amplification due to measurement of $\hat{q}$. The initial state is an eigenstate $\left|q_{0}\right\rangle$ of $\hat{q}$. These are the same trajectories as given in Fig. 2, showing a decreasing relative variance with time $\tau$ and total gain $G$.

One can readily verify, by substitution, that the marginal distributions of the $Q$-function are the unique solutions to these equations. These are equivalent to the stochastic equations

$$
\begin{gathered}
\frac{d q}{d \tau_{-}}=-q+d w_{1}, \\
\frac{d p}{d \tau}=-p+d w_{2},
\end{gathered}
$$

where the noise terms have correlations given by

$$
\left\langle d w_{\mu}(\tau) d w_{\nu}\left(\tau^{\prime}\right)\right\rangle=2 \delta_{\mu \nu} \delta\left(\tau-\tau^{\prime}\right) .
$$

Typical trajectory solutions to the stochastic equations for $q(\tau)$ with $q_{0}=1$ are given in Fig. 2 as a function of dimensionless time. The corresponding results for $q_{m}=q / G$ are given in Fig. 3, showing how the inferred or measured result $q_{m}$ converge to the exact eigenvalue, $q_{0}=1$, as the total gain increases with time. These results agree with the distributions in Fig. 1 for the marginal distributions of $q_{m}$, calculated either using the pure state solutions $\hat{\rho}(t)$ or the $Q$-function equations.

\section{Measurement of a discrete eigenvalue}

We next consider measurement of a discrete eigenvalue, as typified by a two-level system or qubit. Suppose the qubit is in a superposition state, $|\psi\rangle_{q}=(|\uparrow\rangle+|\downarrow\rangle) / \sqrt{2}$. This is equivalent to a spin $1 / 2$ state, with a Pauli spin operator $\hat{\sigma}_{z}=|\uparrow\rangle\langle\uparrow|-| \downarrow\rangle\langle\downarrow|$. The outcome of measuring $\hat{\sigma}_{z}$ is either 1 or -1 . To model the measurement of $\hat{\sigma}_{z}$, we employ a commonly used experimental Hamiltonian [87-89]:

$$
H_{M}=\frac{\hbar g}{2} \hat{n} \hat{\sigma}_{z} .
$$

The measurement is performed by coupling the qubit to an optical field. The field is a single mode with boson operator $\hat{a}$ and number operator $\hat{n}=\hat{a}^{\dagger} \hat{a}$. The optical meter field is 
prepared in a coherent state $|G / i\rangle_{c}$ and coupled for a time $t_{m}$. Thus, the coupled initial state is

$$
|\psi(0)\rangle_{q m}=(|\uparrow\rangle+|\downarrow\rangle)|G / i\rangle_{c} / \sqrt{2} .
$$

Here $G$ is the gain of the meter. This approach is even applicable in the limit of $G \rightarrow 0$, where it yields the large fluctuations found in weak measurement theory [87,90]. However, we are interested in the macroscopic or large $G$ limit, so that vacuum fluctuations are relatively negligible. With an input superposition incident on the measurement device, the final measured state after evolution for a dimensionless measurement time of $\tau_{m}=\pi$, where $\tau=g t$, is

$$
\left|\psi\left(\tau_{m}\right)\right\rangle_{q m}=\frac{1}{\sqrt{2}}\left[|\uparrow\rangle|G\rangle_{c}+|\downarrow\rangle|-G\rangle_{c}\right] .
$$

This describes the final state of the two-mode system and the final state of the meter field. An observation is then made of the real quadrature $x$ of the meter field, regarded as an element of reality.

Here the stochastic description includes a spin degree of freedom, which can be treated either using a fermionic [52] or $\mathrm{SU}(2)$ atomic coherent state [31,91] $Q$-function. The simplest method uses the $\mathrm{SU}(2)$ atomic coherent state $|z\rangle_{a}$, defined as

$$
|z\rangle_{a}=\frac{1}{\sqrt{1+|z|^{2}}} \exp \left(z \hat{\sigma}^{+}\right)|\downarrow\rangle,
$$

where $\hat{\sigma}^{+}=|\uparrow\rangle\langle\downarrow|$ is the qubit raising operator. The normalized basis for the spin $Q$-function is then

$$
\hat{\Lambda}_{f}(z)=\frac{2}{\pi\left(1+z z^{*}\right)^{2}}|z\rangle\left\langle\left. z\right|_{a} .\right.
$$

In this case, the coupled $Q$-function is given by

$$
Q(\alpha, z)=\operatorname{Tr}\left[\hat{\rho} \hat{\Lambda}_{f}(z) \hat{\Lambda}_{b}(\alpha)\right] .
$$

The spin differential identity for the Pauli spin operator $z-$ component is

$$
\hat{\sigma}_{z}|z\rangle\left\langle\left. z\right|_{a}=\left[2 z \partial_{z}+\frac{z z^{*}-1}{1+z z^{*}}\right] \mid z\right\rangle\left\langle\left. z\right|_{a} .\right.
$$

The interaction Hamiltonian is cubic in the operators. To transform this into differential form, it is convenient to transform to logarithmic variables and use basis operators $\hat{\Lambda}_{\eta}$, $\hat{\Lambda}_{\phi}$ normalized in a logarithmic space defined so that

$$
\begin{gathered}
z=e^{\eta}=e^{\eta^{\prime}+i \eta^{\prime \prime}}, \\
\alpha=e^{\phi}=e^{\phi^{\prime}+i \phi^{\prime \prime}} .
\end{gathered}
$$

Using these variables, and introducing $m(\eta)=$ $(3 / 2) \tanh \left(\eta^{\prime}\right), \quad n(\phi)=e^{2 \phi^{\prime}}-1$, one obtains simpler identities, such that

$$
\begin{aligned}
\hat{\sigma}_{z} \hat{\Lambda}_{\eta} & =2\left[\partial_{\eta}+m(\eta)\right] \hat{\Lambda}_{\eta}, \\
\hat{n} \hat{\Lambda}_{\phi} & =\left[\partial_{\phi}+n(\phi)\right] \hat{\Lambda}_{\eta} .
\end{aligned}
$$

After making use of the differential identities, and transforming to logarithmic variables, one obtains that $\partial_{\tau} \dot{Q}=$ $i \operatorname{Tr}\left[\hat{\rho}\left(\hat{\sigma}_{z} \hat{n} \hat{\Lambda}-\hat{\Lambda} \hat{\sigma}_{z} \hat{n}\right)\right] / 2$. After substituting the identities, this gives a generalized Fokker-Planck equation with constant

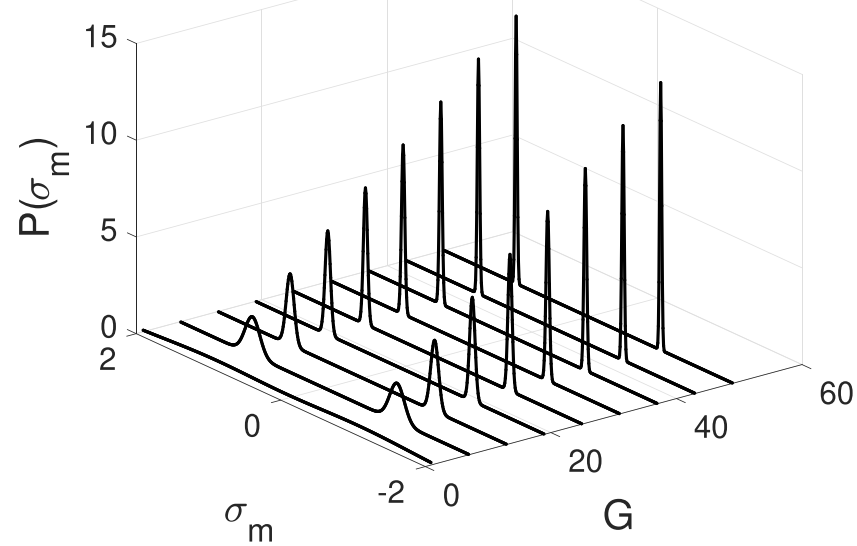

FIG. 4. Probability density $P\left(\sigma_{m}\right)$ for a spin measurement, as a function of the measured spin projection $\sigma_{m}$ and measurement gain $G$. The increasing sharpness with gain demonstrates the measurement outcome for a discrete spin observable. The two peaks correspond to the two possible spin projections.

diffusion, of the traceless form given in Eq (9):

$$
\partial_{\tau} Q=\left[\partial_{\eta^{\prime \prime}} n+\partial_{\phi^{\prime \prime}} m+i\left(\partial_{\phi} \partial_{\eta}-\partial_{\phi}^{*} \partial_{\eta}^{*}\right)\right] Q .
$$

This has a similar behavior to the earlier gain equations, and leads to a $Q$-function solution corresponding to Eq (26). Therefore, the same general type of forward-backward stochastic equations are obtained as previously. We note that there is a symmetric physical explanation of each term. The drift term in the qubit phase, $\eta^{\prime \prime}$, is driven by the meter occupation number $n$, while the drift term in the meter phase, $\phi^{\prime \prime}$, is driven by the qubit occupation number $m$. The remaining terms describe quantum noise and are symmetric in both the meter and qubit.

To analyze the observations or meter readings in detail, we define a measured spin projection as the observed measurement result from the meter, where the spin projection is inferred from the $q$ quadrature:

$$
\sigma_{m}=\frac{q}{G} \text {. }
$$

Observations on the meter only require the output probability for the stochastic field, which at time $\tau=\tau_{m}$ is given by

$$
\begin{aligned}
Q\left(\alpha, \tau_{m}\right) & =\operatorname{Tr}\left[\hat{\rho}\left(\tau_{m}\right) \hat{\Lambda}_{b}(\alpha)\right] \\
& =\frac{1}{2 \pi}\left[e^{-|G-\alpha|^{2}}+e^{-|G+\alpha|^{2}}\right] .
\end{aligned}
$$

The resulting probability distributions, as they develop in time, are shown in Fig. 4 for different gains $G$. This plots $P\left(\sigma_{m}\right)$, the probability of a measured value $\sigma_{m}$ obtained from the $Q$-function for $q$, after integrating over the transverse coordinate $p$, where

$$
P\left(\sigma_{m}\right)=\frac{G}{2 \sqrt{\pi}}\left[e^{-G^{2}\left|\sigma_{m}-1\right|^{2}}+e^{-G^{2}\left|\sigma_{m}+1\right|^{2}}\right] .
$$

We have shown that a $Q$-function phase-space coordinate has a distribution that becomes relatively sharp after a highgain measurement. This allows an observer to determine 
which trajectory is the objectively real one, and hence to calculate with a reduced phase-space ensemble. This corresponds to an "epistemic," or information based, projection of $\hat{\rho}$. For $G$ large, the two different values \pm 1 for $\hat{\sigma}$ can also be measured by the different sign of the outcomes for $\sigma_{m}$ or $q$, using a binning method to reduce the effects of quantum noise.

\section{E. Bell inequality violations}

The strongest objections to realistic interpretations are through correlated measurements that violate Bell inequalities, which have been studied previously using both $Q-$ and $P$-function methods [92-94]. We now consider correlated, spatially separated spins in the Bell state [17],

$$
|\psi\rangle=\left(|\uparrow\rangle_{A}|\downarrow\rangle_{B}-|\downarrow\rangle_{A}|\uparrow\rangle_{B}\right) / \sqrt{2} \text {. }
$$

In the experiment, four different types of correlated measurements are made for spins $\sigma_{\theta}^{A}$ and $\sigma_{\phi}^{B}$ in the $\theta$ and $\phi$ directions, and the results compared. We assume that the eigenvalue relationships are such that

$$
\begin{aligned}
& \hat{\sigma}_{\theta}^{A}|\uparrow\rangle_{\theta}^{A}=|\uparrow\rangle_{\theta}^{A}, \\
& \hat{\sigma}_{\theta}^{A}|\downarrow\rangle_{\theta}^{A}=-|\downarrow\rangle_{\theta}^{A} .
\end{aligned}
$$

The Clauser-Horne-Shimony-Holt Bell inequality is that, for a local hidden variable model of quantum mechanics, given four different correlations $E\left(\theta_{i}, \phi_{j}\right)=\left\langle\hat{\sigma}_{\theta_{i}}^{A} \hat{\sigma}_{\phi_{j}}^{B}\right\rangle$ of spin, one must have [95-97]

$$
B=E\left(\theta_{1}, \phi_{1}\right)-E\left(\theta_{1}, \phi_{2}\right)+E\left(\theta_{2}, \phi_{2}\right)+E\left(\theta_{2}, \phi_{1}\right) \leqslant 2 .
$$

We can now use the stochastic $Q$-function for a calculation of these four measured correlations, by extending the phase space of the previous subsection, now with four complex variables. Each different correlation corresponds to a different measurement Hamiltonian, namely,

$$
H(\theta, \phi)=\hbar g\left[\hat{\sigma}_{\theta}^{A} \hat{n}^{A}+\hat{\sigma}_{\phi}^{B} \hat{n}^{B}\right] .
$$

In this case, one has that the coupled initial state, including the meter states at the two locations, is

$$
|\psi(0)\rangle_{q m}=\frac{1}{\sqrt{2}}\left(|\uparrow\rangle_{A}|\downarrow\rangle_{B}-|\downarrow\rangle_{A}|\uparrow\rangle_{B}\right)|G / i\rangle_{A}|G / i\rangle_{B} .
$$

Here $G$ is the gain of each meter as previously. The final measured state after evolution under a measurement time of $t_{m}=\pi / 2 g$ is

$$
\begin{aligned}
\left|\psi\left(t_{m}\right)\right\rangle_{q m}= & \frac{1}{\sqrt{2}}|\uparrow\rangle_{A}|\downarrow\rangle_{B}|G\rangle_{A}|-G\rangle_{B} \\
& -\frac{1}{\sqrt{2}}|\downarrow\rangle_{A}|\uparrow\rangle_{B}|-G\rangle_{A}|G\rangle_{B} .
\end{aligned}
$$

With the view that the phase-space coordinates of the meter are elements of reality, we can calculate the observed correlations using the procedure outlined above for observing the spin orientation from the output sign of the meter quadratures. The four correlations are obtained from the inferred spins through a process of binning each phase-space coordinate to give a binary estimate of the measured spin, $\sigma_{b}=\operatorname{Sgn}\left(\sigma_{m}\right)$. We find that the Bell violation is a function of the gain, and is given by $B=2 \sqrt{2} \eta^{2}(G)$, where $\eta(G)=$

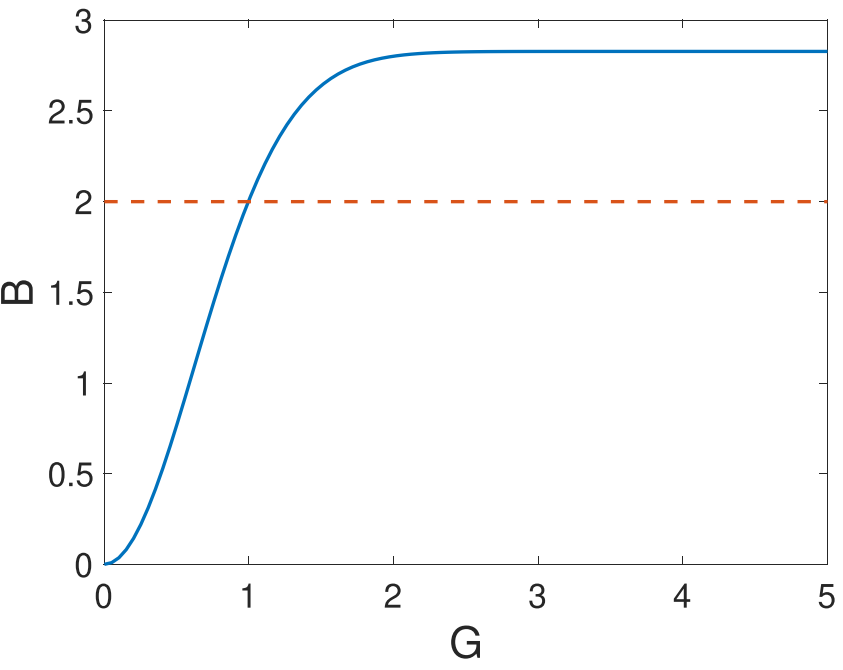

FIG. 5. Measured Bell correlation $B(G)$ obtained from the $Q$ function for a spin-measurement outcome, using a binned inferred spin projection $\sigma_{b}$ and measurement gain $G$. An inferred Bell violation is obtained for gains $G \gtrsim 1$.

$\frac{1}{2}[\operatorname{erf}(G)+1-\operatorname{erfc}(G)]$ is the binning efficiency, which is reduced at low gain. The resulting Bell violation is plotted in Fig. 5.

A Bell violation of $B>2$ is obtained for high enough efficiency, and $G>1$ is already enough to observe this.

\section{F. Phase space and macroscopic realism}

Macroscopic interpretations can be gained from other quantum phase-space methods [14]. The Wigner function is positive-in fact, it becomes the $Q$-function - with the addition of Gaussian noise at the level of Planck's constant, which is macroscopically negligible. This gives an interpretation for a macroscopic superposition state, after interaction with an amplifying meter, in terms of a macroscopic hidden variable, as explained in Refs. [14,98]. Consider a superposition of two position eigenstates, or a superposition of two coherent states with real amplitudes $q_{1}$ and $q_{2}$, so that a measurement of the position quadrature $\hat{q}$ gives two macroscopically distinct outcomes, $q_{1}$ and $q_{2}$. The existence of a positive phase-space distribution means that a macroscopic hidden variable exists, to determine the outcome of the measurement of the quadrature $q$ to be either $q_{1}$ or $q_{2}$, to within an uncertainty of order Planck's constant. The two outcomes are distinguishable for $q_{1}$ and $q_{2}$ when macroscopically distinct. A similar interpretation is given for the entangled cat-state,

$$
|\psi\rangle=\frac{1}{\sqrt{2}}[|\uparrow\rangle|\alpha\rangle+|\downarrow\rangle|-\alpha\rangle],
$$

where here $\alpha$ is large and real, and we take $q_{1}=\alpha$ and $q_{2}=-\alpha$. The Wigner or $Q$-function for this state gives a description that is consistent with the validity of a macroscopic local hidden variable, that predetermines the outcome of the measurement of quadrature $\hat{q}$ to be either $q_{1}$ or $q_{2}$, since the nonpositive nature of the Wigner distribution is small in the macroscopic limit.

In this way, without decoherence, the system can be viewed as being "in one state or the other," where by "states" one 
simply refers to a description predetermining the outcome to be either $q_{1}$ or $q_{2}$. Hence, one has an interpretation in which the system is not paradoxically in "both states at once," which gives a partial resolution of the measurement or Schrödinger cat paradox. A paradox remains, however, at the microscopic level (of order $\hbar$ ), in that the system cannot be viewed as being in one or other of two quantum states, that determine the system to be $q_{1}$ or $q_{2}$ [99-101].

The reality model presented in this paper is consistent with the above interpretation, but deepens and extends it, suggesting that the time-evolution of the complex stochastic variable of the $Q$-function corresponds to a trajectory for an individual "element of reality" at all times. Thus, for example, if the original quantum state in our measurement example were a mixture or eigenstates of $q$ rather than a superposition, one would see little difference in the $q$ trajectories under the gain dynamics, but the $p$ trajectories would differ microscopically. In this approach, the distribution is related to knowledge or epistemology, while the phase-space trajectory is ontological, or real even at the microscopic level. The epistemological nature of the probability distribution means that an observer may decide that a measurement gives them an improved knowledge of the distribution. This Bayesian inference has no direct effect on the objective trajectories.

If there are reservoir couplings to the system, decoherence is introduced. Quantum mechanics predicts that the reduced density matrix for a superposition of eigenstates $\left|q_{1}\right\rangle$ and $\left|q_{2}\right\rangle$ evolves into a mixture of $\left|q_{1}\right\rangle$ and $\left|q_{2}\right\rangle$. That is, the reduced system-meter density matrix after decoherence corresponds to a probabilistic mixture of the two eigenstates. The corresponding reduced $Q$-function describes an ensemble of system-meter pairs. The trajectories of the mixture differ from those of the superposition in that, while the trajectories for $q$ are similar, the trajectories for the complementary observable $p$ are different. However, in the standard quantum approach, an additional nonunitary projection is needed to have just one outcome. This is not necessary in our interpretation. A single macroscopic outcome is a natural consequence of having one realistic trajectory at all stages, both microscopic and macroscopic, and this requires neither decoherence nor wave-function collapse.

\section{QUANTUM PARADOXES}

There are many apparent contradictions between what quantum mechanics predicts and what one might expect in an ontological model. In this section the most well-known paradoxes are discussed briefly. We explain how these are resolved, at least in principle. Since the conventional interpretation has a long history, we cannot treat all known issues here, although further detailed analysis will be carried out elsewhere. Hence, the discussion given below is brief, with an emphasis on conceptual points. We summarize possible objections and paradoxes in this interpretation of quantum mechanics, and their resolution, as follows:

\section{A. Quantum fluctuations}

Since $Q$-functions are statistical, including vacuum fluctuations, how can they represent eigenstates of measurements?
In a full representation of physical reality, the measuring device should be included. Examples are treated above, and in each case the vacuum fluctuations are suppressed relative to the measurement outcome, by a factor equal to the overall gain. Thus, the final measurement outcome becomes sharp. It corresponds precisely to the amplified value of the eigenvalue, after accounting for the gain, for a system initially in an eigenstate of the observable being measured.

\section{B. The Einstein-Podolsky-Rosen (EPR) paradox}

Given the EPR argument [10,102], surely this must be a local hidden variable theory? The "elements of reality" given in our model are not local hidden variables in the sense defined by EPR. This is shown in Sec. IV E by the violation of a Bell inequality. The dynamics of the $Q$-function requires diffusive propagation in a backward time direction. Correlations permitted in retrocausal propagation are inconsistent with EPR's local realism postulates, but they are allowed here. This can be analyzed through the parametric interaction [76] used to demonstrate the EPR argument, and will be treated elsewhere.

\section{Schrödinger's cat and entanglement}

How does this resolve Schrödinger's question [7], that a state such as Eq. (26) after the measurement suggests a macroscopic object in two places $q_{1}$ and $q_{2}$ at once, like a cat simultaneously "living and dead?" This issue arises in the measurement problem, where the measured system becomes entangled with the meter, as we describe in Sec. IV. The explanation is that the $Q$-function evolves as the system interacts with the measurement device. The scaled projection along $p$ at any given time is plotted in Fig. 4. At high enough gain, so that $q_{1}$ and $q_{2}$ are macroscopically separated, this gives a definite result compatible with quantum predictions, and consistent with the pointer being either at $q_{1}$ or $q_{2}$, as explained in Refs. [14,98]. The cat is either living or dead, but not both.

\section{Quantum to classical transition}

Does this explain macroscopic classical behavior? The superposition state of Eq. (43) is consistent with macroscopic reality, since for large $\alpha$, the result of the measurement of $\hat{x}$ is for any individual system given by a well-defined stochastic field quadrature value. Cat-states of this type arise as a result of the measurement process, which amplifies microscopic superposition states into macroscopic (entangled) ones. This occurs when a macroscopic device is coupled to a smaller system. Our model provides an explanation of the measurement problem in which the quantum-to-classical transition takes place via amplification, rather than decoherence.

Decoherence is significant in the longer term, because any coupling to a reservoir introduces loss of information about the entangled system. This brings about an irreversible dynamics. In our model, however, macroscopic realism holds prior to decoherence.

This picture also explains why there is such sensitivity of a macroscopic superposition state to a very small amount of decoherence. It is known that the macroscopic superposition state collapses more quickly, or is more 
sensitive to decoherence, as the size of the separation $\left|q_{1}-q_{2}\right|$ increases [1]. The explanation of this in terms of the macroscopic reality interpretation given by phase-space methods, and also in terms of the "elements of reality" of the cat-states, was suggested in [14,98]. However, our detailed microscopic model gives a more precise explanation in terms of trajectories. As $\alpha$ increases, the phase-space trajectories for $q$ become indistinguishable from those of a classical mixture of the states $\left|q_{1}\right\rangle$ and $\left|q_{2}\right\rangle$. Differences occur for the trajectories in the complementary observable $p$, but these become close to that of the mixture, as $\alpha$ increases. Thus, the sensitivity to decoherence can be viewed as the underlying dynamics for the quantum state becoming close to that of the mixture, as $\alpha$ increases, and hence more readily disrupted by the reservoir couplings.

\section{E. Bell's theorem and causality}

Bell's theorem [17] proves that if one attempts to complete quantum mechanics with local hidden variables, there will be a contradiction with quantum experiments [103-108]. We have shown that Bell violations are obtained using phasespace variables as elements of reality, if one includes the measurement. It is already known from absorber theory $[25,27,28]$, that future time boundary conditions can cause violation of Bell inequalities. A similar backward time propagation occurs in $Q$-function dynamics [31]. One can have locality, with no information transfer [109], while violating the Bell inequality. In the multipartite case, up to 60 simultaneous spatially separated measurements with genuine multipartite entanglement and nonlocality have been simulated using these methods [94]. Due to vacuum fluctuations, a model of the measurement is needed for Bell violations and "all-or-nothing" effects [110].

\section{F. Particle statistics}

Husimi $Q$-functions are defined for bosons, yet the physical world includes fermions. Research on fermionic Gaussian operators [56] shows that fermionic $Q$-functions exist $[52,111]$ with a real and positive distribution. This complete phase space is based on the bounded homogeneous spaces of group theory [58,59]. Any fermionic operator expectation value and its dynamics [71] can be computed, in a similar way to the Husimi $Q$-function. While we have used simpler SU(2) coherent state projectors in this paper, these can be generalized to Fermi fields.

\section{G. Uniqueness}

Why should coherent state projectors represent macroscopic realism, as opposed to other measurement operators? Ontological models should not depend on how measurements are implemented. Coherent state projectors provide a minimal, unbiased implementation of the group symmetry of the field commutators in the standard model. Their role was recognized by Schrödinger [112] and others [53] who proved that they have classical behavior at the macroscopic level.

\section{H. Delayed choice}

The measurement problem is also related to delayed choice experiments [113]. This idea was proposed by Wheeler, who was also responsible (with Feynman) for absorber theory, which was an early attempt at understanding future boundary conditions in electrodynamics. The fact that boundary conditions in the future propagate retrocausally in amplifiers provides an elegant explanation of why choice of measurement can be made at a later time. This will be treated in detail elsewhere.

\section{Contextuality}

Does a $Q$-function model have the contextuality properties of quantum mechanics [114]? It achieves this because the model includes interactions with measuring devices. This was also the nature of Bohr's response to the EPR paradox [18]. When there is a new context, that is, a different measuring device, the measured result is changed through retrocausal interactions. This is completely different to that occurring in a classical, hidden variable model, of the type that is used to analyze contextuality in the Kochen-Specker theorem. Contextuality is not unexpected in a model of reality, since the context, or measurement, is part of the physical world.

\section{SUMMARY}

We have shown that a realistic ontological model for quantum mechanics is obtainable by utilizing a phase space of stochastic fields. The $Q$-function gives the probability of a given field configuration as a generalized phase-space coordinate. Vacuum fluctuations are suppressed in measurements with gain, for both continuous and discrete eigenvalue examples. After the gain is included, sharp eigenvalues are obtained. Thus, the fields can represent an objective physical reality, without an explicit collapse on measurement.

The coherent states are complete and give a unique set of "addresses" in the quantum world, with positive probability. There is also an exact mapping from unitary quantum field dynamics to $Q$-function dynamics, which is obtained from the operator identities. This uses the fact that quantum field Hamiltonians are at most quartic in the quantum fields. Quantum dynamics can therefore be re-expressed as differential equations that have a time-symmetric action principle and path integral.

Our discussion of measurement focuses on idealized cases, just as in most discussions of measurement, because the goal is to understand how the intrinsically noisy $Q$-function distribution can describe an idealized measurement, leading to a well-defined eigenvalue. One can certainly include noise and decoherence to make even more realistic models. We do not do this here, as it tends to obscure the fundamental issue, which is that measurement involves gain or amplification. Gain provides a mechanism by which intrinsic microscopic vacuum noise is relatively suppressed.

The main thrust of this paper was to show how the inclusion of a meter allows one to obtain well-defined eigenvalues in amplified measurements. This is an important fundamental issue, since the stochastic fields themselves have quantum fluctuations, and a well-defined eigenvalue is not compatible 
with large fluctuations in the measured result. $Q$-function dynamical equations, as we have shown, give exactly the required properties. Quantum noise is reduced through retrocausal propagation in the amplified quadrature of a low-noise measuring device. Since only one of the two complementary quadratures of a field can be amplified in this way, the physics of measurement enforces the uncertainty principle.

The $Q$-function dynamical equations for unitary evolution have a traceless diffusion, with equal positive and negative diffusion terms, leading to retrocausal effects from boundary conditions in the future. Related phenomena in electrodynamics were studied by Tetrode, Dirac, Wheeler, and Feynman $[25,27]$, which Dirac called a "beautiful" aspect of his theory. Here, negative diffusion terms occur in parametric amplifier equations, and are directly responsible for the sharp measurement results given above. As a result, Bell's arguments about local hidden variable theories do not apply to $Q$-functions. The model has a different type of time-evolution to a hidden variable theory. One can study more complicated effects, and include decoherence if necessary, but this is not essential to the development of measurement results: the only required ingredient is the meter itself, as indeed one might expect physically.

In summary, we demonstrate the existence of an objective, relativistically invariant ontology for quantum fields.

\section{ACKNOWLEDGMENTS}

P.D.D. and M.D.R. thank the generous hospitality of ITAMP at Harvard University, the Weizmann Institute of Science, and the Joint Institute of Laboratory Astrophysics of The University of Colorado. This work was funded through Australian Research Council Discovery Project Grants No. DP180102470 and No. DP190101480, and performed in part at Aspen Center for Physics, which is supported by National Science Foundation Grant No. PHY-1607611.

\section{APPENDIX: INTERPRETATIONS OF QUANTUM MEASUREMENT}

There are a variety of previous interpretations of quantum mechanics which are reviewed in several places $[43,115,116]$. Without giving a complete survey, we will provide a compressed summary of most earlier approaches, and explain how they differ from our proposal.

We will start with Leggett's classification of three common strands in quantum measurement interpretations [117], noting that some interpretations combine more than one of these ideas. These three common ideas are the statistical interpretation, many worlds, and the decoherence approach. We then consider three approaches which add additional structure to the usual Hilbert space, namely, the Bohm theory of pilot-waves, the spontaneous collapse idea, and an alternative phase-space interpretation.

\section{The statistical interpretation}

The original Copenhagen theory of measurement proposed that quantum mechanics only gives information about the statistics of measurements on an ensemble of identically prepared experiments $[50,118]$. Thus, it provides no ontological interpretation of the microscopic events that cause the statistics. The founder of this approach was Bohr, whose measurement interpretation is in almost all standard textbooks [8]. Several later viewpoints share this approach of a statistical interpretation. The most well-known are QBism [119] and consistent histories [36], although they have other features as well. In its original form, the statistical interpretation is unable to explain how a measurement differs from ordinary unitary evolution, and what is the underlying reality that the statistical theory describes. It is particularly silent on what happens between measurements, or in other words, "is the moon there when nobody looks?" [120].

\section{The many-universe interpretation}

This interpretation [41] regards the Hilbert space statevector as objectively real. It sees no contradiction in the proposal that the universe "really" is a macroscopic superposition of many possibilities. In this approach observers are not aware of other universes. The explanation of how and why observers become unaware of all the other universes, and which of these are to be regarded as 'real', is a difficulty with many universe theory. This approach has been criticized as not properly accounting for space-time properties [121]. A variant of this uses multiple interacting classical universes [122], although in a restricted, nonrelativistic context.

What we propose is a model of reality as a single universe. The fact that many realizations are possible is a different thing to claiming that they all exist simultaneously. In this sense our proposal is akin to statistical mechanics, which also allows for many possible realizations, while only requiring one to exist. A theory of one universe is more parsimonious than a many-universe theory. A picture of just one universe seems preferable according to Occam's razor [123], as requiring fewer assumptions. It leads to a picture of reality as objective fields that exist in space-time, which is a simpler physical understanding of "what is."

\section{The decoherence interpretation}

This approach focuses on the fact that macroscopic states decohere, or become highly entangled with their environment. As Bell [43] and Leggett [117] have emphasized, this still leaves open the question of how just one of the resulting macroscopic states emerges as the preferred alternative during measurement. Decoherence interpretations do not provide a mechanism to project out an individual eigenstate. This requires a nonunitary step in conventional quantum mechanics. As a result, Leggett, Bell, and others have questioned whether decoherence actually solves the measurement problem.

The projection issue is apparently avoided if decoherence is combined with a many-universe interpretation, as in quantum Darwinism [40,124], and other related approaches. It might be argued that this approach leads to a lack of parsimony, pointed out above, and there are known technical objections [125] as well.

In our stochastic field approach, decoherence also occurs whenever a system is in contact with a reservoir. This is a 
normal part of quantum evolution. However, the question of whether an observable is macroscopic, that is, large enough for a macroscopic observer to register it, requires amplification. Therefore, we regard the amplification step as fundamental to measurement. Decoherence occurs in many situations, but gain is essential to having macroscopic or "classical" measurement results, as pointed out by Bohr in his discussions with Einstein at Solvay [48]. In the $Q$-function model, decoherence will create irreversibility in a measurement, if it is present, but it is not required to explain having a definite outcome.

\section{Pilot-wave theory}

In the Bohm theory [9], particles all move in a potential that depends on the wave-function. This formulation, in its original version, has no quantum fields. While there are extensions of this approach to treat quantum field theories [126], not all aspects of quantum fields can be treated in a pilot-wave approach as yet. The stochastic field approach we propose is directly based on quantum field theory, and uses fields as its ontological elements. It has no wave-function requirement, since the $Q$-function dynamics can be worked out without having a wave-function. A Hilbert space or quantum state can be used if convenient, but is not fundamental.

\section{Spontaneous collapse proposals}

Another proposed route to solve measurement problems is the use of additional mechanisms outside of quantum mechanics. These may involve either a random collapse with no currently known physical origin [44], or a mechanism involving gravity $[45,46]$. In general, the hallmark of these approaches is that there is an extra parameter with an unknown magnitude. While such processes may exist, they are not necessary in the present interpretation of reality. Extensions to treat additional decoherence including gravity in a $Q$-function realism model are certainly possible. However, they do not appear essential.

\section{Other phase-space ontologies}

There is another phase-space interpretation of quantum measurement [35], using a different approach. This uses nonrelativistic quantum mechanics, and considers ontic variables with an epistemological restriction of the order of Planck's constant. It has distributions rather than actual trajectories as elements of realism. Since it is not based on fields, it is unclear whether it can reproduce the levels of current precision of quantum field theory. Hydrogenlike spectra are calculated and measured to 12 decimals [127], and heliumlike spectra to 6 decimals [128], all of which require a full theory of QED for their accurate prediction.
[1] M. Brune, E. Hagley, J. Dreyer, X. Maitre, A. Maali, C. Wunderlich, J. M. Raimond, and S. Haroche, Phys. Rev. Lett. 77, 4887 (1996).

[2] J. D. Teufel, T. Donner, M. Castellanos-Beltran, J. W. Harlow, and K. W. Lehnert, Nat. Nanotechnol. 4, 820 (2009).

[3] S. Touzard, A. Grimm, Z. Leghtas, S. O. Mundhada, P. Reinhold, C. Axline, M. Reagor, K. Chou, J. Blumoff, K. M. Sliwa et al., Phys. Rev. X 8, 021005 (2018).

[4] S. Coleman, Phys. Rev. D 15, 2929 (1977).

[5] C. G. Callan and S. Coleman, Phys. Rev. D 16, 1762 (1977).

[6] A. R. Liddle and D. H. Lyth, Cosmological Inflation and Large-Scale Structure (Cambridge University Press, Cambridge, UK, 2000), p. 400.

[7] E. Schrödinger, Naturwissenschaften 23, 823 (1935).

[8] P. A. M. Dirac, The Principles of Quantum Mechanics (Oxford University Press, Oxford, UK, 1981).

[9] D. Bohm, Phys. Rev. 85, 166 (1952).

[10] A. Einstein, B. Podolsky, and N. Rosen, Phys. Rev. 47, 777 (1935).

[11] J. S. Bell, Rev. Mod. Phys. 38, 447 (1966).

[12] A. J. Leggett and A. Garg, Phys. Rev. Lett. 54, 857 (1985).

[13] C. Emary, N. Lambert, and F. Nori, Rep. Prog. Phys. 77, 016001 (2013).

[14] M. D. Reid, J. Phys. A: Math. Theor. 50, 41LT01 (2017).

[15] E. P. Wigner, Am. J. Phys. 31, 6 (1963).

[16] S. Weinberg, E=Einstein: His Life, His Thought, and His Influence on Our Culture (Sterling Publishing, New York, 2006), p. 309.

[17] J. S. Bell, Physics 1, 195 (1964).

[18] N. Bohr, Phys. Rev. 48, 696 (1935).
[19] S. Saunders, J. Barrett, A. Kent, and D. Wallace, Many Worlds?: Everett, Quantum Theory, \& Reality (Oxford University Press, Oxford, 2010).

[20] N. Harrigan and R. W. Spekkens, Found. Phys. 40, 125 (2010).

[21] M. F. Pusey, J. Barrett, and T. Rudolph, Nat. Phys. 8, 475 (2012).

[22] J. Barrett, E. G. Cavalcanti, R. Lal, and O. J. E. Maroney, Phys. Rev. Lett. 112, 250403 (2014).

[23] M. Ringbauer, B. Duffus, C. Branciard, E. G. Cavalcanti, A. G. White, and A. Fedrizzi, Nat. Phys. 11, 249 (2015).

[24] M. Leifer, Quanta 3, 67 (2014).

[25] H. M. Tetrode, Z. Phys. 10, 317 (1922).

[26] P. Dirac, Proc. R. Soc. London 167, 148 (1938).

[27] J. A. Wheeler and R. P. Feynman, Rev. Mod. Phys. 17, 157 (1945).

[28] D. T. Pegg, Eur. J. Phys. 3, 44 (1982).

[29] D. T. Pegg, Phys. Scr. 1986, 14 (1986).

[30] D. T. Pegg and S. M. Barnett, J. Opt. B: Quant. Semiclass. Opt. 1, 442 (1999).

[31] A. Altland and F. Haake, Phys. Rev. Lett. 108, 073601 (2012).

[32] E. G. Beltrametti and S. Bugajski, J. Phys. A: Math. Gen. 28, 3329 (1995).

[33] S. Aaronson, Phys. Rev. A 71, 032325 (2005).

[34] R. W. Spekkens, Phys. Rev. A 75, 032110 (2007).

[35] A. Budiyono and D. Rohrlich, Nat. Commun. 8, 1306 (2017).

[36] R. B. Griffiths, J. Stat. Phys. 36, 219 (1984).

[37] D. Dieks, Ann. Phys. 500, 174 (1988).

[38] H. F. Dowker and J. J. Halliwell, Phys. Rev. D 46, 1580 (1992).

[39] M. Gell-Mann and J. B. Hartle, Phys. Rev. D 47, 3345 (1993). 
[40] R. Blume-Kohout and W. H. Zurek, Phys. Rev. A 73, 062310 (2006).

[41] H. Everett, Rev. Mod. Phys. 29, 454 (1957).

[42] A. Einstein, Albert Einstein-Philosopher Scientist (Open Court Publishing, Chicago, 1949), pp. 2-95.

[43] J. S. Bell, Speakable and Unspeakable in Quantum Mechanics: Collected Papers on Quantum Philosophy (Cambridge University Press, Cambridge, UK, 2004).

[44] G. C. Ghirardi, A. Rimini, and T. Weber, Phys. Rev. D 34, 470 (1986).

[45] L. Diosi, Phys. Lett. A 120, 377 (1987).

[46] R. Penrose, Gen. Relativ. Gravit. 28, 581 (1996).

[47] I. Bialynicki-Birula and J. Mycielski, Ann. Phys. 100, 62 (1976).

[48] N. Bohr, Niels Bohr Collected Works, Vol. 7 (Elsevier, Amsterdam, 1996), pp. 339-381.

[49] N. Bohr, Essays 1958-1962 on Atomic Physics and Human Knowledge, Niels Bohr: Philosophical writings Vol. 3 (Ox Bow Press, Woodbridge, 1987).

[50] M. Born, Science 122, 675 (1955).

[51] L. E. C. Rosales-Zárate and P. D. Drummond, J. Phys. A 46, 275203 (2013).

[52] L. E. C. Rosales-Zárate and P. D. Drummond, J. Phys. A 17, 032002 (2015).

[53] R. J. Glauber, Phys. Rev. 131, 2766 (1963).

[54] A. M. Perelomov, Commun. Math. Phys. 26, 222 (1972).

[55] J. F. Corney and P. D. Drummond, Phys. Rev. Lett. 93, 260401 (2004).

[56] J. F. Corney and P. D. Drummond, J. Phys. A 39, 269 (2006).

[57] R. R. Joseph, L. E. Rosales-Zárate, and P. D. Drummond, J. Phys. A: Math. Theor. 51, 245302 (2018).

[58] É. Cartan, Abh. Math. Sem. Univ. Hamburg, Vol. 11 (Springer, Berlin, 1935), pp. 116-162.

[59] L.-K. Hua, Harmonic Analysis of Functions of Several Complex Variables in the Classical Domains (American Mathematical Society, Providence, RI, 1963).

[60] A. Montina, Phys. Rev. Lett. 97, 180401 (2006).

[61] J. Paavola, M. J. W. Hall, M. G. A. Paris, and S. Maniscalco, Phys. Rev. A 84, 012121 (2011).

[62] S. Kochen and E. P. Specker, The Logico-Algebraic Approach to Quantum Mechanics (Springer, Berlin, 1975), pp. 293-328.

[63] E. P. Wigner, Phys. Rev. 40, 749 (1932).

[64] J. E. Moyal, in Mathematical Proceedings of the Cambridge Philosophical Society, Vol. 45 (Cambridge University Press, Cambridge, UK, 1949), pp. 99-124.

[65] P. A. M. Dirac, Rev. Mod. Phys. 17, 195 (1945).

[66] R. P. Feynman, Int. J. Theor. Physical 21, 467 (1982).

[67] K. E. Cahill and R. J. Glauber, Phys. Rev. 177, 1882 (1969).

[68] P. D. Drummond and C. W. Gardiner, J. Phys. A: Math. Gen. 13, 2353 (1980).

[69] K. E. Cahill and R. J. Glauber, Phys. Rev. A 59, 1538 (1999).

[70] J. F. Corney and P. D. Drummond, Phys. Rev. B 73, 125112 (2006).

[71] R. R. Joseph, L. E. C. Rosales-Zárate, and P. D. Drummond, Phys. Rev. A 98, 013638 (2018).

[72] K. Husimi, Proc. Phys. Math. Soc. Jpn. 22, 264 (1940).

[73] P. D. Drummond, arXiv:1910.00001 (2019).

[74] R. Graham, Z. Phys. B 26, 281 (1977).
[75] Y.-C. Liang, Y.-H. Yeh, P. E. Mendonça, R. Y. Teh, M. D. Reid, and P. D. Drummond, Rep. Prog. Phys. 82, 076001 (2019).

[76] M. D. Reid, Phys. Rev. A 40, 913 (1989).

[77] H. P. Yuen, Phys. Rev. A 13, 2226 (1976).

[78] D. F. Walls, Nature 306, 141 (1983).

[79] P. D. Drummond and M. Hillery, The Quantum Theory of Nonlinear Optics (Cambridge University Press, Cambridge, UK, 2014).

[80] R. E. Slusher, B. Yurke, P. Grangier, A. LaPorta, D. F. Walls, and M. Reid, J. Opt. Soc. Am. B 4, 1453 (1987).

[81] H. Vahlbruch, M. Mehmet, K. Danzmann, and R. Schnabel, Phys. Rev. Lett. 117, 110801 (2016).

[82] M. Malnou, D. A. Palken, L. R. Vale, G. C. Hilton, and K. W. Lehnert, Phys. Rev. Appl. 9, 044023 (2018).

[83] C. M. Caves, Phys. Rev. D 23, 1693 (1981).

[84] V. B. Braginsky, F. Y. Khalili, and K. S. Thorne, Quantum Measurement (Cambridge University Press, Cambridge, UK, 1992).

[85] W. L. Power, S. M. Tan, and M. Wilkens, J. Mod. Optics 44, 2591 (1997).

[86] K. B. Wharton and N. Argaman, Rev. Mod. Phys. 92, 021002 (2020).

[87] L. Rosales-Zárate, B. Opanchuk, and M. D. Reid, Phys. Rev. A 97, 032123 (2018).

[88] A. Blais, R.-S. Huang, A. Wallraff, S. M. Girvin, and R. J. Schoelkopf, Phys. Rev. A 69, 062320 (2004).

[89] A. Wallraff, D. I. Schuster, A. Blais, L. Frunzio, R.-S. Huang, J. Majer, S. Kumar, S. M. Girvin, and R. J. Schoelkopf, Nature 431, 162 (2004).

[90] Y. Aharonov, D. Z. Albert, and L. Vaidman, Phys. Rev. Lett. 60, 1351 (1988).

[91] F. Arecchi, E. Courtens, R. Gilmore, and H. Thomas, Phys. Rev. A 6, 2211 (1972).

[92] L. Rosales-Zárate, B. Opanchuk, P. D. Drummond, and M. D. Reid, Phys. Rev. A 90, 022109 (2014).

[93] P. D. Drummond, B. Opanchuk, L. E. C. Rosales-Zárate, and M. D. Reid, Phys. Scr. T160, 014009 (2014).

[94] M. D. Reid, B. Opanchuk, L. Rosales-Zárate, and P. D. Drummond, Phys. Rev. A 90, 012111 (2014).

[95] J. F. Clauser, M. A. Horne, A. Shimony, and R. A. Holt, Phys. Rev. Lett. 23, 880 (1969).

[96] J. F. Clauser and A. Shimony, Rep. Prog. Phys. 41, 1881 (1978).

[97] N. Brunner, D. Cavalcanti, S. Pironio, V. Scarani, and S. Wehner, Rev. Mod. Phys. 86, 419 (2014).

[98] M. D. Reid, arXiv:1604.00623 (2016).

[99] M. D. Reid, Phys. Rev. Lett. 84, 2765 (2000).

[100] M. D. Reid, Phys. Rev. A 100, 052118 (2019).

[101] M. D. Reid, Phys. Rev. A 97, 042113 (2018).

[102] M. D. Reid, P. D. Drummond, W. P. Bowen, E. G. Cavalcanti, P. K. Lam, H. A. Bachor, U. L. Andersen, and G. Leuchs, Rev. Mod. Phys. 81, 1727 (2009).

[103] D. M. Greenberger, M. A. Horne, and A. Zeilinger, in Bell's Theorem, Quantum Theory and Conceptions of the Universe, edited by M. Kafatos (Springer, Berlin, 1989), p. 348.

[104] N. D. Mermin, Phys. Rev. Lett. 65, 1838 (1990).

[105] M. Ardehali, Phys. Rev. A 46, 5375 (1992).

[106] A. V. Belinsky and D. N. Klyshko, Phys. Lett. A 176, 415 (1993). 
[107] D. Collins, N. Gisin, S. Popescu, D. Roberts, and V. Scarani, Phys. Rev. Lett. 88, 170405 (2002).

[108] G. Svetlichny, Phys. Rev. D 35, 3066 (1987).

[109] M. Hillery and B. Yurke, Quantum Semiclassical Opt.: J. Eur. Opt. Soc. Part B 7, 215 (1995).

[110] D. M. Greenberger, M. A. Horne, and A. Zeilinger, Bell's Theorem, Quantum Theory and Conceptions of the Universe (Springer, Berlin, 1989), pp. 69-72.

[111] R. R. Joseph, L. E. C. Rosales-Zárate, and P. D. Drummond, Phys. Rev. A 100, 012337 (2019).

[112] E. Schrödinger, Naturwissenschaften 14, 664 (1926).

[113] J. A. Wheeler, Mathematical Foundations of Quantum Theory (Elsevier, Amsterdam, 1978), pp. 9-48.

[114] S. Kochen and E. P. Specker, J. Math. Mech. 17, 59 (1967).

[115] A. C. Elitzur, S. Dolev, and N. Kolenda, Quo Vadis Quantum Mechanics? (Springer Science \& Business Media, Berlin, 2006).

[116] M. Schlosshauer, Rev. Mod. Phys. 76, 1267 (2005).

[117] A. J. Leggett, Quo Vadis Quantum Mechanics? (Springer, Berlin, 2005), pp. 83-90.
[118] L. E. Ballentine, Rev. Mod. Phys. 42, 358 (1970).

[119] C. A. Fuchs, N. D. Mermin, and R. Schack, Am. J. Phys. 82, 749 (2014).

[120] N. D. Mermin, Phys. Today 38, 38 (1985).

[121] D. Wallace and C. G. Timpson, Br. J. Philos. Sci. 61, 697 (2010).

[122] M. J. W. Hall, D.-A. Deckert, and H. M. Wiseman, Phys. Rev. X 4, 041013 (2014).

[123] J. Schaffer, Australas. J. Philos. 93, 644 (2015).

[124] W. H. Zurek, Phys. Rev. D 24, 1516 (1981).

[125] R. E. Kastner, Stud. Hist. Philos. Sci. Part B: Stud. Hist. Philos. Mod. Phys. 48, 56 (2014).

[126] W. Struyve, Rep. Prog. Phys. 73, 106001 (2010).

[127] A. Beyer, J. Alnis, K. Khabarova, A. Matveev, C. G. Parthey, D. C. Yost, R. Pohl, T. Udem, T. W. Hänsch, and N. Kolachevsky, Ann. Phys. 525, 671 (2013).

[128] C. T. Chantler, M. N. Kinnane, J. D. Gillaspy, L. T. Hudson, A. T. Payne, L. F. Smale, A. Henins, J. M. Pomeroy, J. N. Tan, J. A. Kimpton, E. Takacs, and K. Makonyi, Phys. Rev. Lett. 109, 153001 (2012). 